\title{
Ionised Hydrogen at Large Galactocentric Distances
}

\author{
J. Bland-Hawthorn \\ Anglo-Australian Observatory, PO Box 296, Epping, NSW 2121, Australia \\ jbh@aaossz.aao.gov.au \\ Received 1996 October 14, accepted 1996 December 22
}

\begin{abstract}
We summarise recent attempts to detect warm ionised gas at large galactocentric distances. This includes searches for gas at the edges of spirals, in between cluster galaxies, towards extragalactic $\mathrm{H}_{\mathrm{I}}$ clouds, and towards high-velocity clouds and the Magellanic Stream in the Galaxy. With the exception of extragalactic $\mathrm{H}_{\mathrm{I}}$ clouds, all of these experiments have proved successful. Within each class, we have only observed a handful of objects. It is premature to assess what fraction of the missing baryonic mass fraction might be in the form of ionised gas. But, in most cases, the detections provide a useful constraint on the ambient ionising flux, and, in the case of spiral edges, can even trace dark matter haloes out to radii beyond the reach of radio telescopes.
\end{abstract}

Keywords: galaxies: kinematics and dynamics — dark matter: cosmic radiation field: galactic radiation field

\section{Introduction}

The nature of 'dark matter' is widely recognised as a fundamental astrophysical problem (Carr 1994). Evidence for 'missing' dark matter exists on all scales from star clusters to the Universe itself. The current trend is to suspect that the dark matter is mostly non-baryonic in nature (Hegyi \& Olive 1986), but our current understanding of Big Bang nucleosynthesis indicates that we have only observed about one-tenth of the ordinary baryonic matter in the Universe. This has encouraged speculation that the 'missing baryonic mass' is in the form of cold gas (Pfenniger, Combes \& Martinet 1994) or hot gas (Barcons, Fabian \& Rees 1991). In our view, not enough attention has been paid to the possibility that the missing mass is in the form of warm ionised gas $\left(\sim 10^{4} \mathrm{~K}\right)$ smoothly distributed on scales much larger than the optical disks of galaxies. Such gas, whether photoionised or collisionally ionised, could conceivably be detected by the Fabry-Perot 'staring' technique. This was the original motivation for a three-year campaign with HIFI at the CFHT 3.6 $\mathrm{m}$ telescope, and TAURUS-2 at the AAT $3.9 \mathrm{~m}$ and WHT $4.2 \mathrm{~m}$ telescopes.

We present here a brief summary of results to date. It is premature to assess the likely mass fraction concealed in ionised gas. Instead, we find that detections of ionised gas at large galactocentric distances have important applications to galaxy dynamics and to probing the ambient radiation field.

\section{Intergalactic Gas}

How much cold or warm debris is out there? Searches for intergalactic $\mathrm{H}$ i to date have been discouraging.
Systems like the Magellanic Stream have only been seen in one other galaxy system (Schneider et al. $1983,1989)$, although tidal tails have been traced out to great distances in a number of objects (Hibbard \& Mihos 1995). The only bona fide extragalactic $\mathrm{H}$ I cloud is the H I $1225+01$ SW complex (Giovanelli \& Haynes 1989); the NE cloud is now known to have a weak optical counterpart. Other claims have not been supported by subsequent observations (e.g. Taylor et al. 1995). The situation will no doubt improve with the Parkes Multibeam and Arecibo Drift Scan surveys over the next few years, in that they are expected to find cloud masses as small as $10^{6-7} M_{\odot}$ out to $z \leq 0 \cdot 1$.

There have been various attempts to detect $\mathrm{H}_{\mathrm{I}}$ structures at large galactocentric radius in optical emission lines. Reynolds et al. (1986) achieved a marginal detection of the Leo Ring in $\mathrm{H} \alpha$ but this has not been confirmed by subsequent studies. The Magellanic Stream was marginally detected $(2 \cdot 7 \sigma)$ by the TAURUS-2 group (Bland-Hawthorn, Freeman \& Quinn 1994, unpublished) in their observations of the Sculptor group. A problem that remains unresolved is whether some fraction of this emission arises from clouds within the background cluster, a controversy which began with the original $\mathrm{H}_{\mathrm{I}}$ survey (Haynes \& Roberts 1979; Arp 1985). A more concerted campaign has detected ionised gas in clouds MS II-IV along the stream (Weiner \& Williams 1996); this result is discussed Bland-Hawthorn \& Maloney (1997). No detections in optical emission lines have been achieved in the direction of the extragalactic $\mathrm{H}_{\mathrm{I}}$ clouds, either towards the Haynes-Giovanelli Cloud (q.v. Vogel et al. 1995) or the putative NGC 3256 
cloud (McCain et al. 1995). These observations are discussed in Section 4.

There have been claims for ionised gas from $\mathrm{H}$ I bridges between galaxies, e.g. by Donahue, Aldering \& Stocke (1995), using on-band/off-band subtraction methods. It is questionable whether such methods can be used to make reliable detections at such faint flux levels (emission measures $\varepsilon_{m} \leq 1$ ) as they are extremely susceptible to scattered light. Furthermore, it is important to disperse the light spectrally to avoid any additional contribution from the background (e.g. galaxy, night sky). In particular, the Reynolds Layer [N II] emission, which is transmitted by their filter, is much brighter than the anticipated extragalactic signal. Since the Reynolds Layer is variable and detectable over the entire sky, it could easily provide an excess signal when on- and off-band fields are differenced. This could explain why the claimed $\mathrm{H} \alpha$ distribution closely resembles the galaxy continuum distribution.

Is there evidence for $\mathrm{H}^{+}$clouds between galaxies? The only such system claimed to date is the 'Ant' cloud in Fornax (Bland-Hawthorn et al. 1995). This was originally found from its depolarisation signature towards the huge radio lobes in NGC 1316. We determined that the $10 \mathrm{kpc}$ cloud has a mass of roughly $10^{8} M_{\odot}$ and that it must be almost fully ionised. Follow-up $\mathrm{HI}$ surveys with the VLA have revealed nothing (van Gorkom 1995, private communication). There are many similar depolarisation 'drop outs' across the lobes which might conceivably correspond to a large population of ionised gas clouds. Follow-up observations have been planned to test this hypothesis.

An exciting claim was made by Williams \& Schommer (1993) that they had detected ionised gas from a nearby absorption-line system observed towards $3 \mathrm{C} 273$, even while $\mathrm{H}$ I had not been found in this direction. Indeed, we were encouraged by this result in our efforts to find intergalactic $\mathrm{H}^{+}$gas. Unfortunately, subsequent work has been unable to confirm this claim (Bland-Hawthorn et al. 1994; Vogel et al. 1995).

\section{What is the Fabry-Perot 'Staring' Technique?}

To our knowledge, there are four groups actively engaged in extragalactic work using the 'staring' technique. These programs are based at Wisconsin (since 1971), Rutgers (since 1982), AAO/LPO/Hawaii (since 1985), and Maryland/Caltech (since 1988). Notably, R. J. Reynolds has made use of a $1^{\circ}$ field to characterise the warm ionised gas throughout the Galaxy in $\mathrm{H} \alpha$, [O III], [NII] and [SII]. The other groups typically use fields of view an order of magnitude smaller, which makes them applicable to a wider variety of extragalactic projects than the Wisconsin system.
The 'staring' technique exploits the Jacquinot advantage of the Fabry-Perot to obtain extremely deep spectra of extended, diffuse objects. For a fixed gap spacing, light falling on the instrument with wavelength $\lambda$ is dispersed according to $\lambda \propto \cos \theta$, where $\theta$ is the off-axis field angle. The spectrum in a narrow band is dispersed radially from the optical axis across the field. When the data are binned azimuthally about the optical axis, a single deep spectrum is obtained. As in long-slit spectrometers, the instrumental profile is projected onto the detector but the line FWHM varies across the field according to $\delta \theta \propto \theta^{-1}$. At the AAT, we have already achieved $\mathrm{H} \alpha$ emission measures of $0.2 \mathrm{~cm}^{-6} \mathrm{pc}$ $\left(2 \times 10^{-19} \mathrm{erg} \mathrm{cm}^{-2} \mathrm{~s}^{-1} \operatorname{arcsec}^{-2}\right)$ at the $3 \sigma$ level in about 90 minutes (Bland-Hawthorn et al. 1995). In principle, we are able to reach $0.02 \mathrm{~cm}^{-6} \mathrm{pc}$ $(3 \sigma)$ in about six hours using larger optics. Due to the $\cos \theta$ factor, the raw spectrum has quadratic sampling and needs to be resampled to a linear axis where the original number of bins is preserved.

There are any number of potential pitfalls with the 'staring' technique. To achieve the highest sensitivity, the diffuse source needs to be monochromatic within the detector field and have an intrinsic line dispersion roughly equal to the instrumental profile. A strong kinematic gradient across the field would wash out the signal after azimuthal binning.

There is a wide class of problems relating to the atmosphere. Night sky lines vary in brightness temporally and as a function of airmass. Water absorption features come and go with the site humidity. Subtracting off the background can be particularly hazardous if the 'on' and 'off' spectra are obtained in separate exposures. Using data in the same CCD frame to subtract the background has its own hazards. Thinned chips have a bad fringeing problem at high dispersion. An important test is to show that the spectral baseline is identical in, say, three sectors of the field. Another weakness in many published results to date (e.g. Songaila, Bryant \& Cowie 1989) is that the wavelength domain of the data is typically only 5-10 times the resolution element, a problem well understood by radio astronomers. This greatly complicates defining what constitutes a reliable continuum level as the diffuse emission we are after is usually a faint signal situated between bright night sky lines with dominant wings. For this reason, we have worked hard to achieve $40 \AA$ bandpass at $\mathrm{H} \alpha$ at $1 \AA$ resolution.

For systems at low velocities, we need to contend with both the geocoronal lines and with Galactic emission. Some observers appear to confuse night sky lines with Galactic emission (particularly the [N II] lines), for example. It is possible to lower the background within the resolution element of the expected signal by either using the Earth's motion 
Table 1. Possible sources of truncation in galaxies

\begin{tabular}{lll}
\hline Shape of $\mathrm{H}_{\mathrm{I}}$ disk & \multicolumn{1}{c}{ Source of truncation } & Trend with increasing radius \\
\hline Axisymmetric & Ambient radiation field & $\varepsilon_{m}$ decreasing \\
Bisymmetric & Tides due to external galaxy & $\varepsilon_{m}=0$ \\
Mirror symmetric & Integral-sign warp+internal source & $\varepsilon_{m}$ increasing \\
Asymmetric & Ram pressure from external medium & $\varepsilon_{m}$ complex \\
\hline
\end{tabular}

(i.e. choosing the right time of year to observe) or by observing the object close to local midnight.

\section{Cosmic Ionising Background}

The emission measure $\varepsilon_{m}$ from the surface of a cloud embedded in a bath of ionising radiation gives a direct gauge, independent of distance, of the ambient radiation field beyond the Lyman continuum edge. If we overlook problems of cloud geometry and covering fraction, a truly extragalactic $\mathrm{H}_{\mathrm{I}}$ cloud provides a crucial probe of the metagalactic ionising flux.

The current best $2 \sigma$ upper limit (Vogel et al. 1995) for the cosmic ionising background is $J_{-21}^{0}$ $<0.08\left(\Phi^{0}<3 \times 10^{4}\right.$ phot $\left.\mathrm{cm}^{-2} \mathrm{~s}^{-1}\right) . \quad J_{-21}^{0}$ is the ionising flux density of the cosmic background at the Lyman limit in units of $10^{-21} \mathrm{erg} \mathrm{cm}^{-2}$ $\mathrm{s}^{-1} \mathrm{~Hz}^{-1} \mathrm{sr}^{-1} ; \Phi^{0}\left(=2 \pi J_{-21}^{0} / h\right)$ is the equivalent photon flux at face of a uniform, optically thick slab. For an electron density $n_{\mathrm{e}}$ and an ion density $N_{\mathrm{H}^{+}}$, the expected emission measure from the slab is $\varepsilon_{m}=\int n_{\mathrm{e}} n_{\mathrm{H}^{+}} \mathrm{d} l=n_{\mathrm{e}} n_{\mathrm{H}^{+}} L \mathrm{~cm}^{-6}$ pc where $L$ is the thickness of the ionised region. The resulting emission measure for an ionising flux $\Phi^{0}$ is then $\varepsilon_{m}=1.25 \times 10^{-6} \Phi^{0} \mathrm{~cm}^{-6} \mathrm{pc}$. The upper limit on $J_{-21}^{0}$ is sufficiently deep that it begins to challenge various models for UV background (Haardt \& Madau 1996). While one would certainly want to believe that 'staring' provides a fundamental constraint, there is some uncertainty as to whether the GiovanelliHaynes cloud is a suitable screen for probing the background radiation. The $\mathrm{H}_{\mathrm{I}}$ complex appears to be highly inclined to our line of sight (Chengalur, Giovanelli \& Haynes 1995). What matters is the $\mathrm{H}$ I covering fraction seen by the ionising photons, in other words, along an axis in the plane of the sky.

More recently, we have obtained (McCain et al. 1995) a similar $2 \sigma$ upper limit $\left(J_{-21}^{0}=0.09\right)$ from observations of a putative extragalactic $\mathrm{H}$ I cloud in the NGC 3256 group. This was originally 'discovered' by Jayanne English (1995) but the cloud has yet to be confirmed by follow-up observations. We are wary of Sancisi's dictum that 'most extragalactic $\mathrm{H}_{\mathrm{I}}$ clouds are not confirmed in subsequent observations'.

\section{Ionised Edges of Spiral Galaxies}

Sunyaev (1969) first argued that the HI disks of spiral galaxies truncate at a few times the optical diameter because the exponentially declining $\mathrm{HI}$ column eventually becomes fully ionised by the metagalactic radiation field (see also Bochkarev \& Sunyaev 1975). It was realised by Bland-Hawthorn et al. (1994) that the predicted levels of emission (Maloney 1993; Dove \& Shull 1994) could be reached with the 'staring' technique. However, there may be alternative explanations in specific cases. In particular, Bland-Hawthorn \& Maloney (1997) have shown that the young disk population could be responsible since almost all-if not all-spirals have at least some warp (Kuijken 1996; Carignan 1995, personal communication).

We can summarise possible sources of truncation in galaxies as shown in Table 1. Space does not allow for a discussion of each case. The deprojected shape of the $\mathrm{H}_{\mathrm{I}}$ disk at a fixed column density is shown in the first column. The source of the truncation and the expected trend in $\varepsilon_{m}$ (with increasing radius) are shown in the second and third columns respectively.

In order to explore these cases, we are currently studying a range of galaxy types in different environments. The galaxies chosen for detailed study include M31, M33, M83, NGC 628, NGC 3198, NGC 5266, Fourcade-Figueroa, and the Sculptor group. The most detailed work has concentrated on NGC 253 (Bland-Hawthorn, Freeman \& Quinn 1996), M33 and NGC 3198 (Bland-Hawthorn, Veilleux \& Carignan 1996). In the case of NGC 253, we see ionised gas with $\varepsilon_{m} \sim 0.1 \mathrm{~cm}^{-6}$ at and beyond the $\mathrm{HI}$ disk (Figure 1). The $[\mathrm{N} \mathrm{II}] \lambda 6548 / \mathrm{H} \alpha$ ratio appears to be close to unity. This has enabled us to extend the rotation curve by $25 \%$ over the $\mathrm{H}$ I data. It is unlikely that these ratios can be explained by a quasar-produced ionising background. Such ratios need additional heating without further ionisation, e.g. from ram pressure heating as the disk moves through an external medium. For M33, we have succeeded in detecting $\mathrm{H} \alpha$ at the $\mathrm{H}$ I edge at similar flux levels. The WHT/CFHT observational setups did not allow us to simultaneously observe both $\mathrm{H} \alpha$ and [N II]. This galaxy has a very substantial $\mathrm{HI}_{\mathrm{I}}$ warp and the outer regions may be seeing the central disk population (Patel \& Wilson 1995).

\section{Further Experiments}

The edges of spirals are of intrinsic interest for a number of related experiments. As is well known, the MACHO experiment has shown that maybe as much as half the dark matter out to the distance of the LMC is made up of baryons. One possibility is low-luminosity white dwarfs. In his book, Sciama 


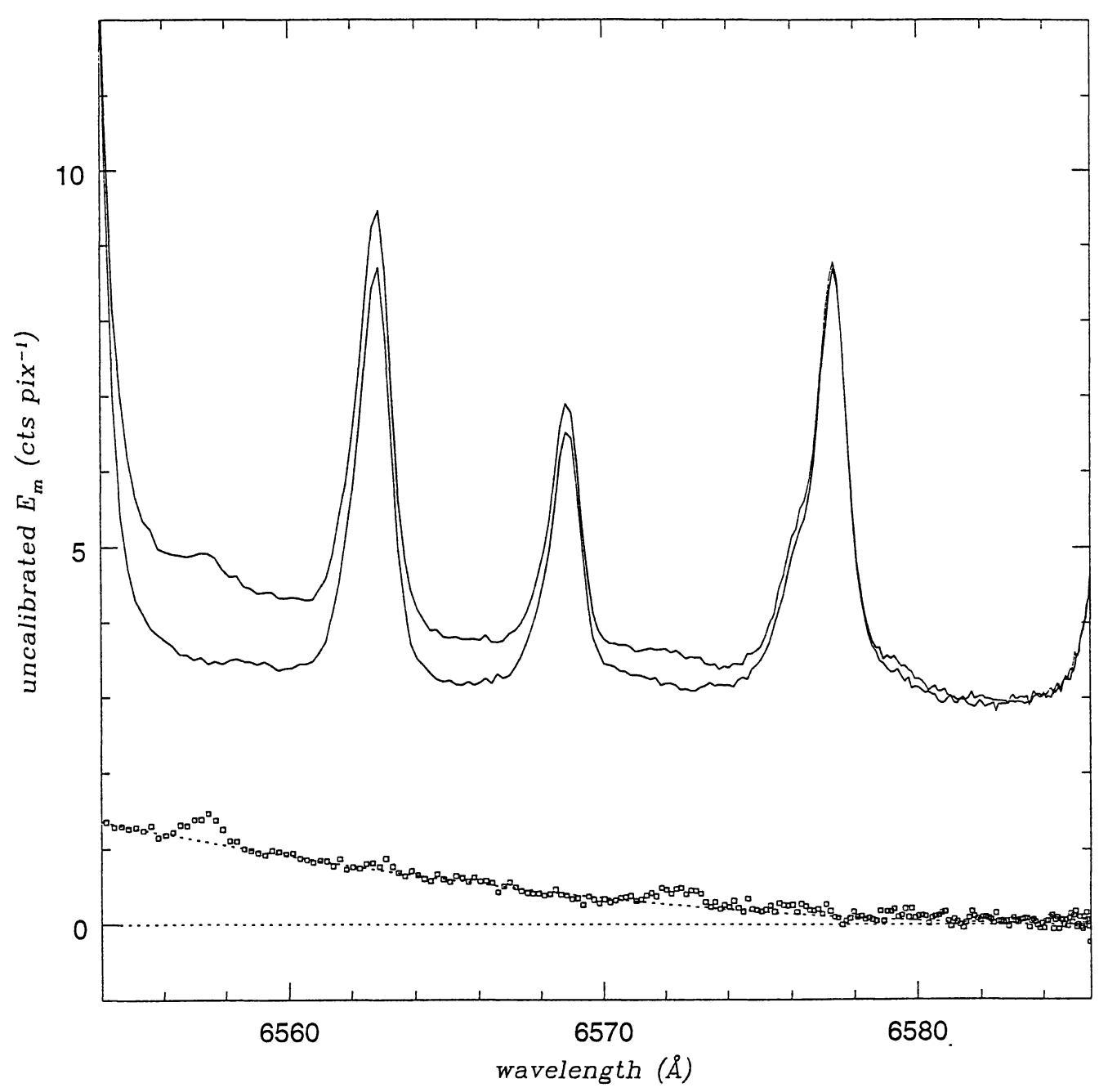

Figure 1-The emission-line spectrum at the H I edge compared with the off-field spectrum. The difference of these spectra is shown below. Remarkably, the [ $\mathrm{N} \mathrm{II}] \lambda 6548$ line has a surface brightness comparable to the $\mathrm{H} \alpha$ surface brightness, as compared with solar-abundance $\mathrm{H}$ II regions where the ratio is an order of magnitude smaller. The azimuthally averaged galaxy continuum underlies the spectrum and corresponds to roughly $\mu_{B}=24 \mathrm{mag} \operatorname{arcsec}^{-2}$ below [N II] falling to $25 \mathrm{mag}$ $\operatorname{arcsec}^{-2}$ below $\mathrm{H} \alpha$.

(1996) demonstrates that tau neutrinos with masses $24 \mathrm{eV}$ and lifetimes of $\sim 10^{23} \mathrm{~s}$ explain a gamut of disjoint observations. Sciama neutrinos share an important similarity with white dwarfs as both could, in principle, make up the dark halo and produce sufficient flux to ionise spiral edges (Sciama 1995).

Once weak line emission is detected, it is very difficult to identify the source of ionisation unambiguously. Thus, we have chosen to study a diverse class of objects in different environments. In other words, to rule out ram pressure from an external medium, we choose galaxies in loosely bound clusters (e.g. Ursa Major). To rule out internal sources of ionisation, we choose ellipticals with extended HI disks (e.g. NGC 5266), but even here there is an uncertain contribution from ' $U V$ upturn' stars. While the early results are promising, we shall refrain from concluding this review with some general statements.

\section{Acknowledgments}

I am grateful to my Australian and French-Canadian colleagues for permission to mention the spiral edge detections. I am indebted to Oxford University for the Visiting Fellowship and for their hospitality while much of this manuscript was drafted. I am deeply indebted to Prof. D. W. Sciama for animated discussions and extensive advice, some of which was acted upon. 
Arp, H. 1985, AJ, 90, 1012

Barcons, X. R., Fabian, A. C., \& Rees, M. J. 1991, Nature, 350,685

Bland-Hawthorn, J., Ekers, R. D., van Breugel, W., Koekemoer, A., \& Taylor, K. 1995, ApJ, 447, L77

Bland-Hawthorn, J., Freeman, K. C., \& Quinn, P. J. 1996, ApJ, submitted

Bland-Hawthorn, J., \& Maloney, P. R. 1997, ApJ, in press

Bland-Hawthorn, J., Taylor, K., Veilleux, S., \& Shopbell, P. L. 1994, ApJ, 437, L95

Bland-Hawthorn, J., Veilleux, S., \& Carignan, C. 1996, in preparation

Bochkarev, N. G., \& Sunyaev, R. A. 1977, Soviet Astr., 21, 542

Carr, B. J. 1994, ARA\&A, 32, 531

Chengalur, J. N., Giovanelli, R., \& Haynes, M. P. 1995, AJ, 109,2415

Donahue, M., Aldering, G., \& Stocke, J. 1995, ApJ, 450, 45

Dove, J., \& Shull, J. M. 1994, ApJ, 423, 196

English, J. 1995, PhD thesis, Australian National University

Giovanelli, R., \& Haynes, M. P. 1989, ApJ, 346, L5

Haardt, F., \& Madau, P. 1996, ApJ, 461, 20

Haynes, M. P., \& Roberts, M. S. 1979, ApJ, 227, 767

Hegyi, D. J., \& Olive, K. A. 1986, ApJ, 303, 56
Hibbard, J. E., \& Mihos, C. J. 1995, AJ, 110, 140

Kuijken, K. 1996, in Dark and Visible Matter in Galaxies and Cosmological Implications

McCain, C., Freeman, K. C., English, J., \& Bland-Hawthorn, J. 1995, in preparation

Maloney, P. R. 1993, ApJ, 414, 41

Patel, K., \& Wilson, C. D. 1995, ApJ, 451, 607

Pfenniger, D., Combes, F., \& Martinet, L. 1994, A\&A, 285, 79

Reynolds, R. J., et al. 1986, ApJ, 309, L9

Schneider, S. E., Helou, G., Salpeter, E. E., \& Terzian, Y. 1983, ApJ, 273, L1

Schneider, S. E., et al. 1989, AJ, 97, 666

Sciama, D. W. 1995, MNRAS, 276, L1

Sciama, D. W. 1996, Modern Cosmology and the Dark Matter Problem, 2nd edn (Cambridge Univ. Press)

Songaila, A., Bryant, W., \& Cowie, L. L. 1989, ApJ, 345, L71

Sunyaev, R. 1969, ApJ Lett., 3, 33

Taylor, C. L., Brinks, E., Grashuis, R. M., \& Skillman, E. D. 1995, ApJS, 99, 427; erratum ApJS, 102, 89

Vogel, S. N., Weymann, R., Rauch, M., \& Hamilton, T. 1995, ApJ, 441, 162

Weiner, B. J., \& Williams, T. B. 1996, AJ, 111, 1156

Williams, T. B., \& Schommer, R. A. 1993, ApJ, 419, L53 